\title{
FACTORES INVOLUCRADOS EN EL PROCESO DE TOMA DE DECISIONES EN BIBLIOTECAS DE TAMAÑO MEDIO Y PEQUEÑO. RESULTADOS DEL PROYECTO DECIMAL
}

\author{
M. Bosch* y C. Espelt*
}

Resumen: Se presentan los resultados de la investigación realizada en el marco del proyecto europeo DECIMAL, que tiene como objetivo el desarrollo de un módulo integrado de soporte de la toma de decisiones para sistemas automatizados usados en bibliotecas pequeñas y medianas. La investigación cuantitativa y cualitativa llevada a cabo en el Reino Unido, Italia y España se ha basado en una combinación de diversos métodos: revisión de la literatura, entrevistas semiestructuradas, cuestionarios y grupos de discusión en ocasión de los dos seminarios de presentación realizados. Se distinguen dos líneas básicas de investigación: la primera en torno a la utilización real de indicadores y medidas para la gestión y evaluación de la actividad del centro, así como su interés potencial en el caso que no se hayan aplicado por el momento, y la segunda en torno al tipo de decisiones más habituales en los centros y los factores que inciden en este proceso (fuentes de información utilizadas, cultura institucional, formación, nivel de satisfacción). El artículo está centrado en los resultados obtenidos en las bibliotecas españolas, aunque se mencionan también los resultados globales a modo de comparación. Las conclusiones del estudio han dado como resultado la especificación de las necesidades de los usuarios, sobre cuya base se ha diseñado el módulo de soporte a la toma de decisiones. El proyecto ha concluido con una fase de evaluación del prototipo que ha implicado el desarrollo de cuatro versiones sucesivas del módulo con la finalidad de resolver los problemas presentados durante el proceso de evaluación.

Palabras clave: evaluación del rendimiento; información para la gestión; proyecto DECIMAL; sistemas de soporte de decisiones; toma de decisiones.

\begin{abstract}
The article presents the research results of the DECIMAL European project. The aim of the project is to produce an integrated desicion support module for library management systems used in small and medium size libraries. The quantitative and qualitative research carried out in United Kingdom, Italy and Spain has been based in a combination of different methods: literature review, interviews, questionnaires and discussion groups during both presentation workshops held. Two basic research lines are discerned: the first one involving the real use of performance measures and indicators in management and evaluation of the library activities, and also their potential interest in the case they have not been applied to the present, and the second one involving the type of decisions most frequantly made in libraries and the issues that influence decision-making process (information sources used, organizational culture, training, satisfaction level). The article has
\end{abstract}

* Escola Universitària de Biblioteconomia i Documentació. Univ. de Barcelona. Correo electrónico: espelt@eudb.ub.es.

Recibido: 28-6-97. 2." versión: 12-1-99. 
been centered in the results obtained in spanish libraries, but the general results are also mentioned in order to be compared. The conclusions of the study have resulted in the user requirement specification, as a first step in the design of the decision support module. The project has concluded with a prototype evaluation phase organised as an iterative testing of the prototypes and successive software modifications. The development of four versions of the module has resolved the problems identified earlier in the evaluation process.

Key words: DECIMAL project; decision support systems; decision-making; management information; performance measurement.

\section{Proyecto DECIMAL}

\subsection{Introducción}

DECIMAL (DECIsion-MAking in Libraries: decision research for the development of integrated library systems) es un proyecto subvencionado por el Programa de Bibliotecas de la Dirección General XIII de la Comisión Europea. Se inició en febrero de 1995 y finalizó en el mes de abril de 1997.

El programa de bibliotecas es una de las siete áreas cubiertas por el programa de aplicaciones telemáticas en el Tercer Programa Marco. DECIMAL forma parte del conjunto de proyectos seleccionados en la tercera convocatoria de noviembre de $1993 \mathrm{den}$ tro de la línea de acción IV, destinada a la estimulación de un mercado europeo de productos y servicios telemáticos específicos para las bibliotecas.

En el tema 18 bis se incluyen los cuatro proyectos relacionados con la investigación y el desarrollo de modelos y herramientas de soporte a la toma de decisiones en las bibliotecas. Además de DECIMAL, se aprobaron los proyectos DECIDE, EQLIPSE y MINSTREL. Partiendo de los datos cuantitativos y, en menor grado, cualitativos que proporcionan los sistemas de automatización en la gestión de los procesos y operaciones de la biblioteca según modelos tradicionales, se pretende llegar a un consenso sobre la forma en que estos datos se deberían producir, explotar e integrar en los procesos de toma de decisiones.

Mientras que DECIMAL orienta su investigación específicamente a bibliotecas pequeñas y medianas, los demás proyectos se dirigen especialmente a bibliotecas universitarias, públicas y nacionales.

\subsection{Objetivos}

La investigación desea potenciar la armonización y convergencia de las políticas nacionales y europeas de bibliotecas desarrollando una especificación de los requerimientos de los usuarios, como base de desarrollo de un estándar para los sistemas de soporte a las decisiones. Tres son los países que participan en el proyecto, lo que ha permitido partir de la expresión de necesidades de las comunidades bibliotecarias de tres ámbitos culturales europeos distintos.

Los objetivos del proyecto pueden agruparse en tres grandes bloques. En primer lugar, se pretende identificar el proceso de toma de decisiones en bibliotecas pequeñas y medianas (bibliotecas médicas, de empresa, de departamentos de la administración 
pública, escolares, de escuelas universitarias, de asociaciones profesionales y de otras instituciones) en los tres países que participan en el proyecto. Se trata de identificar los elementos más significativos de este proceso: los objetivos, el tipo de decisión adoptada y la valoración de sus resultados, las situaciones de decisión predominantes, como la introducción de un nuevo servicio o la evaluación de alguno de los existentes, y los factores que intervienen en el proceso, como aspectos económicos, de estructura interna de la organización, demandas de los usuarios, etc.

El segundo bloque consiste en determinar las necesidades de información de los responsables de las bibliotecas para la toma de decisiones.

Finalmente en tercer lugar, diseñar y desarrollar un módulo de soporte a las decisiones que sea viable comercialmente y que pueda integrarse en los sistemas automatizados de gestión de bibliotecas existentes.

\subsection{Participantes}

DECIMAL ha sido dirigido por el Department of Library and Information Studies de la Manchester Metropolitan University del Reino Unido. Han participado en el proyecto otros dos centros universitarios de biblioteconomía y documentación: el Istituto di Biblioteconomia e Paleografia de la Università degli Studi de Parma (Italia) y la Escola Universitària de Biblioteconomia i Documentació de Barcelona, así como la biblioteca del University College de Oxford y la empresa Inheritance Systems Limited de Oxford.

La distribución de funciones entre los participantes ha venido marcada principalmente por el carácter de cada institución. Los miembros de los centros universitarios han asumido la mayor parte de la realización del proyecto. A partir del diseño inicial del estudio y su planificación, tareas llevadas a cabo por la MMU, se ha trabajado conjuntamente sin una distribución específica de tareas, lo cual ha supuesto un enriquecimiento personal y de los resultados de trabajo, no debido exclusivamente a las aportaciones motivadas por las diferencias nacionales sino a la dinámica de trabajo en equipo. La participación de la responsable de la biblioteca del University College de Oxford a lo largo de todas las fases del proyecto ha aportado la perspectiva de la práctica profesional como contrapunto al carácter académico de la mayoría de los integrantes del equipo; sin embargo su colaboración ha sido más destacada en la evalución del módulo. El personal de Inheritance Systems Ltd. ha centrado su actividad en el diseño y desarrollo inicial del módulo de soporte a la toma de decisiones, así como en las sucesivas modificaciones introducidas para resolver los problemas detectados durante la fase de evaluación.

\subsection{Fases}

La fase de investigación incluye una revisión de la bibliografía sobre gestión de bibliotecas publicada en España, Italia y el Reino Unido, una serie de entrevistas a responsables de bibliotecas seleccionadas y la distribución de dos cuestionarios enviados a una amplia muestra de bibliotecas en los tres países participantes.

A partir de la investigación se ha elaborado la especificación de los requerimien- 
tos de los usuarios, y en base a ella, el proyecto de arquitectura y especificaciones técnicas del módulo de soporte a las decisiones. Una vez finalizado el desarrollo técnico del módulo se ha procedido al test y evaluación del prototipo. Se han programado así mismo sesiones de demostración y evaluación del módulo a responsables de bibliotecas pequeñas y medianas.

\section{Aspectos metodologicos}

\subsection{Métodos empleados para la recogida de datos}

La investigación posterior a la revisión bibliográfica combina diferentes métodos de obtención de datos que permiten contrastar el comportamiento de los profesionales de la información en la gestión del centro y sus necesidades de optimizar el proceso de toma de decisiones.

En primer lugar, se han realizado entrevistas a un grupo de responsables de centros. Se han seleccionado 13 bibliotecas en cada uno de los países, correspondientes a diferentes tipologías. Se han presentado a los entrevistados nueve situaciones de decisión, entre las cuales debían escoger una como hilo conductor de la exposición del caso. El análisis de las entrevistas revela que la técnica del incidente crítico (1), empleada en las entrevistas semiestructuradas, permite expresar libremente ideas y problemas relativos a la situación actual del centro, sin necesidad de previa conceptualización.

El objetivo de las entrevistas es verificar y completar la información obtenida en la revisión de la literatura con la finalidad de diseñar los cuestionarios partiendo de una base sólida. Se han elaborado dos tipos de cuestionarios para recoger información sobre las fuentes de información que se utilizan en la toma de decisiones y el uso de datos estadísticos en la gestión. El cuestionario de información para la toma de decisiones está inspirado en un estudio de McClure (2) centrado en bibliotecas universitarias norteamericanas. El contenido específico de este cuestionario consiste en 20 situaciones de decisión que deben relacionarse con las dos fuentes de información más frecuentemente utilizadas, elegidas de una lista de fuentes. El total de 34 fuentes se agrupa en cinco apartados: documentos publicados, documentos internos, contactos personales, contactos a nivel de grupos y organismos e intuición y experiencia personal.

El uso de datos estadísticos en la gestión de las bibliotecas se ha investigado a partir de un cuestionario en el que se presenta una tabla de 102 medidas e indicadores del rendimiento elaborada por De Monfort University en el marco del proyecto PROLIB (3). Estas medidas e indicadores del rendimiento están presentadas en relación a determinadas áreas de actividad del centro: contexto general de la biblioteca, personal, puntos de servicio y horario de apertura, usuarios, utilización, materiales bibliográficos, servicio de referencia, préstamo interbibliotecario e instalaciones.

Los dos cuestionarios incluyen un conjunto de cuestiones sobre las características del centro - personal, usuarios, colecciones, automatización- así como sobre el nivel de protagonismo y satisfacción de los profesionales respecto a la toma de decisiones.

Los datos obtenidos de las respuestas a los cuestionarios permiten avanzar en el conocimiento del proceso de toma de decisiones, el uso de información para la gestión y el alcance real de la aplicación de las técnicas de evaluación del rendimiento a partir de medidas e indicadores. 
En el transcurso del proyecto se han programado dos seminarios con la finalidad de dar difusión a los resultados del proyecto entre el colectivo profesional de cada país. A pesar del objetivo principal de estas actividades, la información obtenida en las sesiones de debate se ha demostrado de gran utilidad, siendo incorporada a los resultados de las fases anteriores.

\subsection{Muestra de bibliotecas}

La selección de bibliotecas responde a los criterios definidos en el planteamiento básico del proyecto, ya que se trata de uno de los puntos diferenciales respecto al resto de proyectos relativos a la toma de decisiones.

Se pretende centrar el estudio en bibliotecas de dimensiones limitadas. Mientras que la gestión en los grandes centros ha sido ampliamente tratada tanto en la bibliografía como en otros proyectos, el ámbito de las bibliotecas pequeñas y medianas carece de estudios en profundidad. Así mismo, las grandes bibliotecas están representadas por categorías muy bien delimitadas, principalmente públicas, universitarias y grandes bibliotecas para la investigación, mientras que, en el caso de las bibliotecas de dimensiones inferiores, la diversidad es muy amplia y coexisten varias características que dificultan el establecimiento de las tipologías excluyentes. El ámbito de especialización que cubre el centro y la institución de la que depende dan como resultado tipologías no coincidentes.

El sistema bibliotecario de cada país ha influido en la selección de las bibliotecas, ya que el funcionamiento más o menos extendido de redes condiciona el nivel de autonomía en la gestión que llevan a cabo los responsables de cada centro. El ejemplo que ilustra mejor este caso es el de las bibliotecas públicas que ha sido objeto de un tratamiento distinto en cada país. En el Reino Unido no se ha incluido ninguna biblioteca pública, mientras que en España se ha seleccionado un número limitado de públicas que no forman parte de ninguna de las redes existentes y, en Italia, se han contemplado de forma más generalizada, debido a su organización más descentralizada.

La tipología establecida para analizar los datos procedentes de los cuestionarios está formada por bibliotecas escolares, de college (en España se han seleccionado centros universitarios que no forman parte de un sistema), médicas, de asociaciones profesionales, de empresa, de organismos de la administración pública y otras. Los centros seleccionados para las entrevistas responden, igualmente, a la tipología anterior. Sin embargo, la participación en los seminarios de presentación ha sido más heterogénea, debido al interés que suscita este tema entre los profesionales de las grandes bibliotecas.

De un total de 360 encuestas distribuidas, 60 de información para la toma de decisiones y 60 de medición del rendimiento, por país, se han recibido 139 respuestas, 56 de las cuales corresponden a España y, dentro de éstas, 31 provienen de Cataluña. Los porcentajes de respuesta por países son: Reino Unido $55 \%$, España 46,6 \% e Italia $13,3 \%$.

De las respuestas recibidas, un $42 \%$ en España, un $40 \%$ en Italia y un $32 \%$ en el Reino Unido corresponden a bibliotecas con un solo profesional. Destacan las bibliotecas médicas españolas con un $75 \%$. Mientras que el número más elevado de profesionales se presenta en las bibliotecas de la administración pública (de 1 a 12 profesionales). 
Aunque en resultados globales el Reino Unido es el país con el número máximo más bajo de personal ayudante, es en España donde es mayor el porcentaje de centros con un solo ayudante (el $34 \%$ ) y además tres bibliotecas no disponen de personal de esta categoría.

Las respuestas recibidas muestran que el porcentaje de centros que disponen de un sistema de automatización es del $70 \%$ (60\% de los resultados globales). Estos datos responden a las características de la muestra: centros que destacan por su participación activa en la vida profesional y, por tanto, no necesariamente representativos del conjunto. Cabe destacar que, de la extensa lista de programas citados, únicamente uno de ellos, Data Trek, es utilizado en más de un país.

Se mencionan también otros programas dedicados específicamente a la gestión, como programas estadísticos, hojas de cálculo y procesadores de texto; sin embargo su utilización es muy mínima ( $25 \%$ del total global).

De estas características dominantes en la muestra se desprenden algunas de las condiciones que deberá reunir el módulo de soporte a la toma de decisiones: capacidad de llevar a cabo funciones ofimáticas, compatibilidad con programas de automatización muy variados, facilidad y rapidez de aprendizaje y manejo.

\section{Principales resultados}

\subsection{Intervención en la toma de decisiones}

Analizaremos en primer lugar el grado de protagonismo de los profesionales en la toma de decisiones, de acuerdo con los datos obtenidos del conjunto de los cuestionarios.

Ante las opciones de órgano responsable de la toma de decisiones -equipo directivo, comité, jefe de área, bibliotecario, otros y conjuntamente- el $39 \%$ de las respuestas españolas señala el propio bibliotecario, porcentaje sensiblemente inferior al 55 $\%$ del resultado global. Así mismo la participación de más de un órgano o persona en este proceso es más habitual en nuestro país $(37 \%)$ que en el resto según el porcentaje global $(20 \%)$. Los comentarios añadidos por algunos de los encuestados apoyan estas cifras al considerar aconsejable la toma de decisiones compartida. Las razones de esta preferencia se basan en el hecho de proporcionar al bibliotecario una valoración más realista de las necesidades del usuario y de aumentar la comprensión de los servicios bibliotecarios por parte de otros sectores de la organización.

La mayoría de los profesionales se muestra satisfecha con la información de que dispone para la gestión del centro. En una escala de cinco valores, el $67 \%$ se sitúa en los dos niveles más altos y tan sólo un $6 \%$ se inclina por los dos inferiores. Los resultados españoles muestran únicamente una desviación de dos puntos a la baja respecto a los globales.

$\mathrm{Al}$ considerar el nivel de responsabilidad del profesional en la toma de decisiones hay que tener en cuenta cuáles son las situaciones de decisión en las que interviene. El cuestionario de información para la toma de decisiones ha proporcionado datos específicos de responsabilidad en cada una de las 20 situaciones de decisión presentadas.

Los resultados globales confirman los temas más tratados en la bibliografía revisada. Así pues, la selección y compra de materiales bibliográficos es la más frecuen- 
temente decidida por el bibliotecario (en un $70 \%$ de los casos), seguida de la elección del sistema de automatización, la reorganización de las instalaciones y la elaboración del reglamento de la biblioteca. Por el contrario, las decisiones relativas a la subcontratación de servicios, cobro de servicios, selección de personal y traslados son asumidas por otros órganos.

Las diferencias entre ámbitos geográficos no son significativas a excepción de las decisiones relacionadas con la gestión de recursos humanos que, a diferencia del Reino Unido, en España e Italia no corresponden normalmente al bibliotecario.

\subsection{Utilización de indicadores y medidas}

\section{,}

La práctica totalidad de las bibliotecas $(91 \%)$ recopilan estadísticas; no obstante, las respuestas al cuestionario específico dedicado a la medición del rendimiento muestran la aplicación de este tipo de datos a la gestión del centro. El diseño de la encuesta permite hacer la distinción entre datos interesantes para la gestión, según la opinión del bibliotecario, y datos que se utilizan realmente en el centro, ofreciendo cuatro casos distintos para cada una de las 102 medidas e indicadores: utilizado, disponible pero no utilizado, no disponible pero de interés, no interesa. Como ya se ha comentado, la lista se presenta dividida en secciones de acuerdo con las actividades del centro; sin embargo, en la fase de análisis, los datos se han dividido en dos grupos correspondientes a indicadores y medidas, entendiendo por indicador la combinación de dos o más medidas para determinar el rendimiento.

Una primera constatación de este análisis evidencia que el grupo de los indicadores tiene una utilización y un interés inferior al de las medidas, aunque los resultados españoles muestran un porcentaje de utilización de indicadores superior, un $54,2 \%$ frente al $43,3 \%$ en general.

Otro aspecto general en el cual se advierte una actitud diferente es que los bibliotecarios españoles utilizan en menor grado los datos disponibles y, por el contrario, manifiestan un interés superior por medidas e indicadores de los que no disponen en la actualidad.

Los resultados confirman el interés de los profesionales por los materiales bibliográficos, el nivel de satisfacción del usuario con la colección, la información obtenida y las instalaciones y los servicios. En concreto, destaca el interés por medidas aplicadas al préstamo; una gran parte de los centros controla el número total de préstamos realizados y manifiesta interés en obtener el número de documentos no prestados, el de títulos prestados, y el indicador de la proporción de ejemplares en préstamo.

Entre las áreas de menor utilización e interés cabe destacar las medidas e indicadores referidas a personal, con una media de $-48,40 \%$ en España y de $-34,30 \%$ en el conjunto de los tres países; se trata del número de personal y los gastos de este capítulo, ya que uno de los indicadores más utilizados es el tiempo que el personal dedica a su actividad principal. Asimismo, los indicadores per cápita no son utilizados y tampoco tienen ningún interés para los responsables de centros de pequeñas dimensiones.

Según el tipo de biblioteca se detectan diferencias importantes en las medidas de rendimiento que interesan. Dos ejemplos representativos de estas diferencias son las bibliotecas escolares, que muestran su interés casi exclusivamente por los datos relati- 
vos a los servicios de referencia, y las bibliotecas médicas que consideran prioritario el servicio de préstamo interbibliotecario.

\subsection{Factores que inciden en la toma de decisiones}

En vista de los resultados anteriores, es evidente que los profesionales no fundamentan sus decisiones en los datos estadísticos, lo cual hace imprescindible la investigación complementaria sobre las fuentes de información en las que se apoyan.

Los resultados del cuestionario de información para la toma de decisiones demuestran la importancia del elemento personal en este proceso. Las teorías de gestión atribuyen a las relaciones personales más riqueza informacional que a los documentos publicados y los datos numéricos, pero este tipo de datos plantean más dificultades para su integración en un módulo de soporte a la toma de decisiones.

Dentro del conjunto de fuentes de información de carácter personal, en España la propia experiencia de trabajo y la opinión e intuición personal han sido las fuentes que han obtenido un mayor número de respuestas $(20,4 \%)$, seguidas de la opinión del personal bibliotecario, del centro o de otras organizaciones, a nivel individual o en comisiones y grupos de trabajo $(19,2 \%)$. Los contactos con personas de otros departamentos, a nivel individual o en grupos, forman parte también de las fuentes más valoradas, pero el porcentaje es sensiblemente inferior $(7,9 \%)$. En el conjunto de los tres países se consideran más útiles los contactos con personal bibliotecario $(20,7 \%)$, aunque la experiencia y la intuición personal ocupan el segundo lugar $(12,9 \%)$.

El segundo grupo de fuentes destacadas por los bibliotecarios españoles es la documentación interna, principalmente los objetivos de la biblioteca, los informes internos y los datos estadísticos $(16,7 \%)$. Los resultados generales dan más importancia a la información obtenida de los usuarios, ya sea en base a una relación personal o a las encuestas de usuarios $(10,8 \%$, frente a un $6,5 \%$ en España). A nivel comparativo sobresale también la escasa utilización de la literatura profesional (2,9\% España $-5,6 \%$ general). Atribuimos esta diferencia a la dificultad de tener acceso regular a este tipo de material, debido a la falta de presupuesto de los centros pequeños para subscripciones especializadas destinadas únicamente al bibliotecario.

Aunque en la lista de fuentes se incluyeron el correo electrónico y las listas de distribución y las fuentes de información electrónica en línea e Internet, la utilización de este tipo de información electrónica es muy poco significativa. En España es evidente que la comunicación electrónica gana terreno día a día, y por tanto es palpable que la situación ha cambiado desde junio de 1995; sin embargo es necesario tener en cuenta que los centros pequeños no siempre disponen de facilidades para el uso interno de estos recursos.

A partir de los comentarios de los profesionales, en el marco de las sesiones de debate o bien añadidos a los cuestionarios, deben mencionarse otros factores que influyen en la toma de decisiones. En Italia y España aparece con insistencia la falta de formación en técnicas de gestión. Los bibliotecarios son conscientes de no poseer los conocimientos necesarios para enfrentarse a la evaluación de los servicios que ofrece el centro, principalmente la selección de los datos a obtener y su aplicación. Abundan los centros con problemas de falta de personal, especialmente personal administrativo. El bibliotecario debe asumir varias funciones y llevar a cabo todo tipo de trabajos, lo cual 
desemboca en una falta de tiempo para la gestión y la evaluación del rendimiento. La falta de sensibilización de los estamentos directivos hacia los servicios que puede ofrecer la biblioteca a la organización es otra de las dificultades para la gestión del centro.

\section{Especificación de los requisitos del módulo}

La investigación realizada ha permitido un análisis detallado de las necesidades de los usuarios, con la finalidad de identificar los factores involucrados en el proceso de toma de decisiones en las bibliotecas. Las características cualitativas y cuantitativas que desempeñan un papel de ayuda en la toma de decisiones han sido agrupadas por categorias, y se les ha asignado un nivel de prioridad teniendo en cuenta la tipología y tamaño de las bibliotecas, las diferencias de cultura institucional, los datos procedentes del sistema de automatización y otras fuentes externas.

La especificación de los requisitos que deberá reunir el módulo se deduce de este trabajo de síntesis e incluye las siguientes características básicas:

- Facilidad de manejo y aprendizaje; incorporación de mensajes de ayuda sensibles al contexto y un programa de formación que permita resolver dudas sobre la medición del rendimiento.

- Capacidades multilingües.

\section{Figura 1}

Módulo de soporte a la toma de decisiones. Pantalla del menú principal
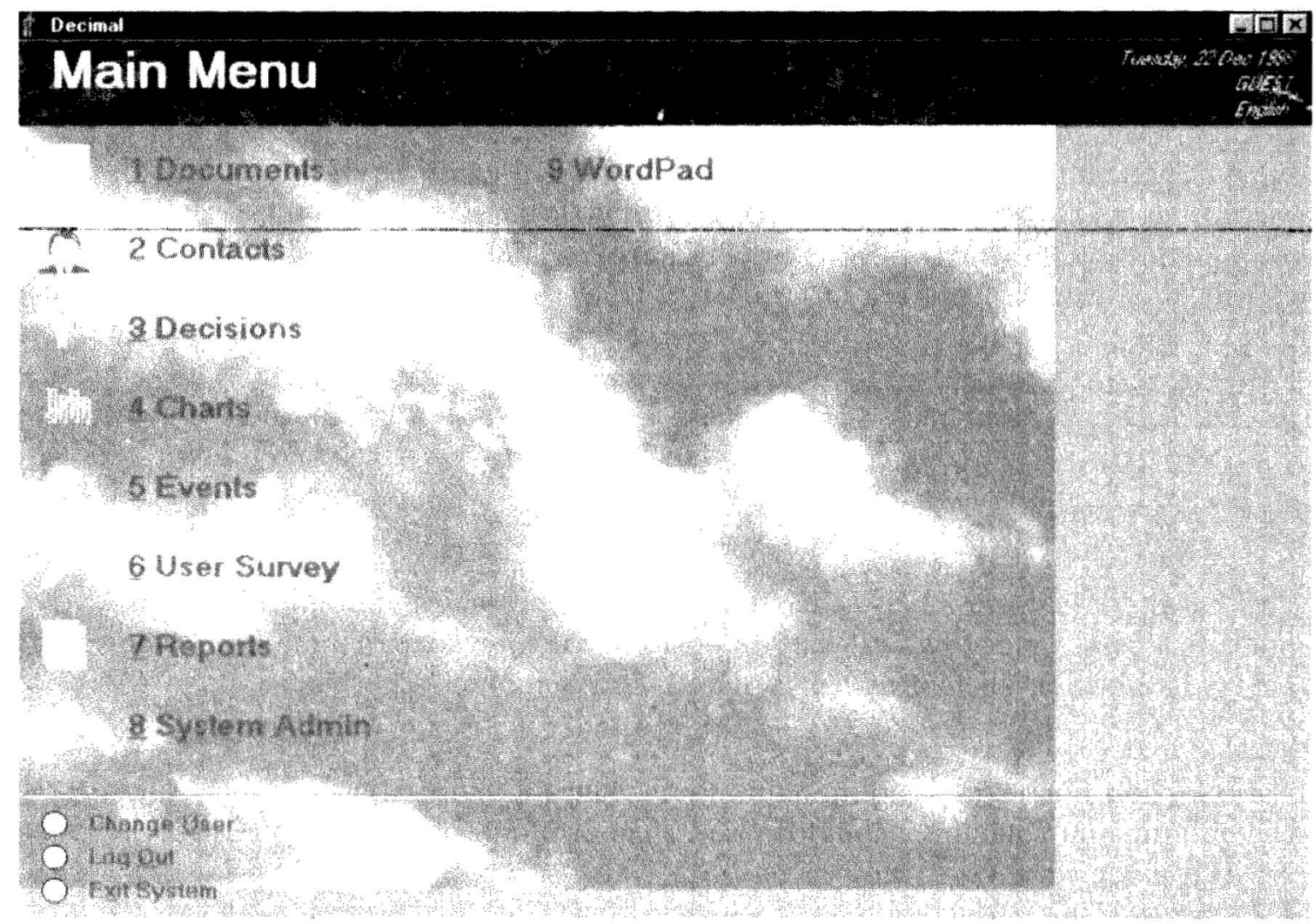

Rev. Esp. Doc. Cient, 22, 1, 194x) 
- Facilidad de adecuación del sistema a las necesidades del centro; flexibilidad para modificar la interfaz.

- Bajo coste.

- Compatibilidad con los sistemas operativos más comunes (DOS y Windows).

- Compatibilidad con sistemas de automatización de bibliotecas y otros sistemas utilizados en las organizaciones, con la finalidad de acceder y transferir datos.

- Integración de información numérica, textual, gráfica, imágenes y voz.

- Integración de los sistemas de comunicación más comunes (corrreo electrónico, Internet).

- Archivo de datos para identificar tendencias a partir de la comparación de datos actuales e históricos.

- Variedad en la forma de presentación de los datos.

- Posibilidad de un proceso interactivo de recopilación de datos (encuestas a usuarios).

- Seguridad de los datos.

Los criterios de evaluación del módulo se han elaborado en base a las características anteriores.

\section{Evaluación del módulo}

En el proceso de evaluación del módulo han intervenido los miembros del equipo del proyecto, especialmente durante una primera etapa de detección de fallos, y algunos bibliotecarios como usuarios potenciales del producto. Esta participación externa se ha concretado en las sesiones de presentación del módulo, estudios de casos y evaluaciones intensivas.

Se han diferenciado dos áreas básicas de evaluación según el tipo de datos: cuantitativos (relativos a medidas e indicadores) y cualitativos, como documentos, notas de trabajo, etc. Asimismo se ha distinguido entre una evaluación subjetiva, centrada en la imagen y la respuesta emocional ante las características del producto, y una evaluación objetiva en base a una lista de 31 criterios elaborada a partir de las características mencionadas en el apartado anterior.

En cada uno de los países se ha realizado una sesión de presentación y demostración del modulo. Las intervenciones de los asistentes en el debate y las respuestas a los cuestionarios distribuidos han permitido obtener algunas conclusiones. Los bibliotecarios valoraron positivamente o muy positivamente el diseño de las pantallas en un $96 \%$ (en España $90 \%$ ). En cuanto a la utilidad, consideraron el módulo útil o muy útil para la biblioteca en un $90 \%$ (80\%), y para otros departamentos de la empresa o institución en un $80 \%(74 \%)$. Los datos estadísticos se consideraron más apropiados que los documentos y fuentes de información, $90 \%$ frente a $84 \%$ (79\% frente a $46 \%$ ).

La opinión general de los usuarios potenciales se ha completado con los estudios de casos llevados a cabo en Italia y España, tres sesiones de carácter individual con responsables de centros de tipologías diferentes, que han permitido obtener valoraciones sobre las diversas funciones del módulo y su utilidad en contextos específicos.

En el Reino Unido, la responsable de la biblioteca del University College de Oxford, miembro del equipo del proyecto, y un bibliotecario con experiencia en medición 
Figura 2

Pantalla del menú de gráficos

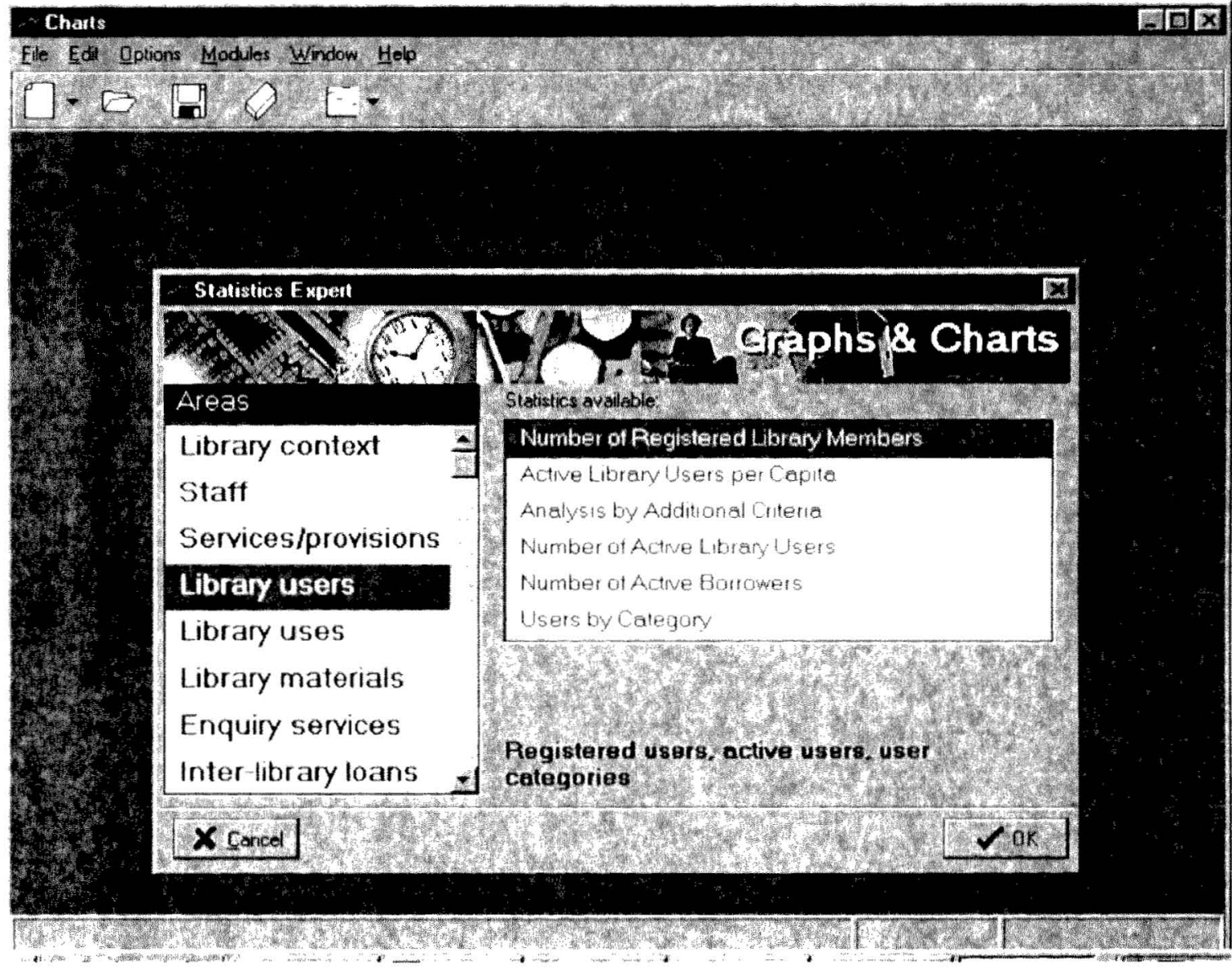

del rendimiento en bibliotecas pequeñas y medianas, contratado para este propósito, han elaborado dos evaluaciones con carácter más exhaustivo.

Resulta imposible resumir en el reducido ámbito de este artículo los resultados de la evaluación detallada; sin embargo se considera importante mencionar una de las limitaciones del prototipo utilizado en la evaluación. La integración de datos procedentes de otras aplicaciones es fundamental para un módulo de soporte a la toma de decisiones. Los bibliotecarios la han valorado mucho en los casos en que se ha podido demostrar, como en el correo electrónico, procesadores de texto y hojas de cálculo. pero ha sido un gran inconveniente el hecho de no poder mostrarla en sistemas de automatización de bibliotecas ya que, aún en el caso de que no la hayan reclamado especificamente, ha influido en su visión global del módulo.

Aunque la cuarta versión del módulo ha sido suficientemente completa para eva luar la mayoría de los objetivos del módulo, no deja de ser un prototipo que requiere un desarrollo adicional para su utilización en contextos externos al ámbito del proyec to. Este desarrollo posterior, así como la posible comercialización del producto, son responsabilidad de Inheritance Systems Limited 
Figura 3

Pantalla de encuesta a los usuarios

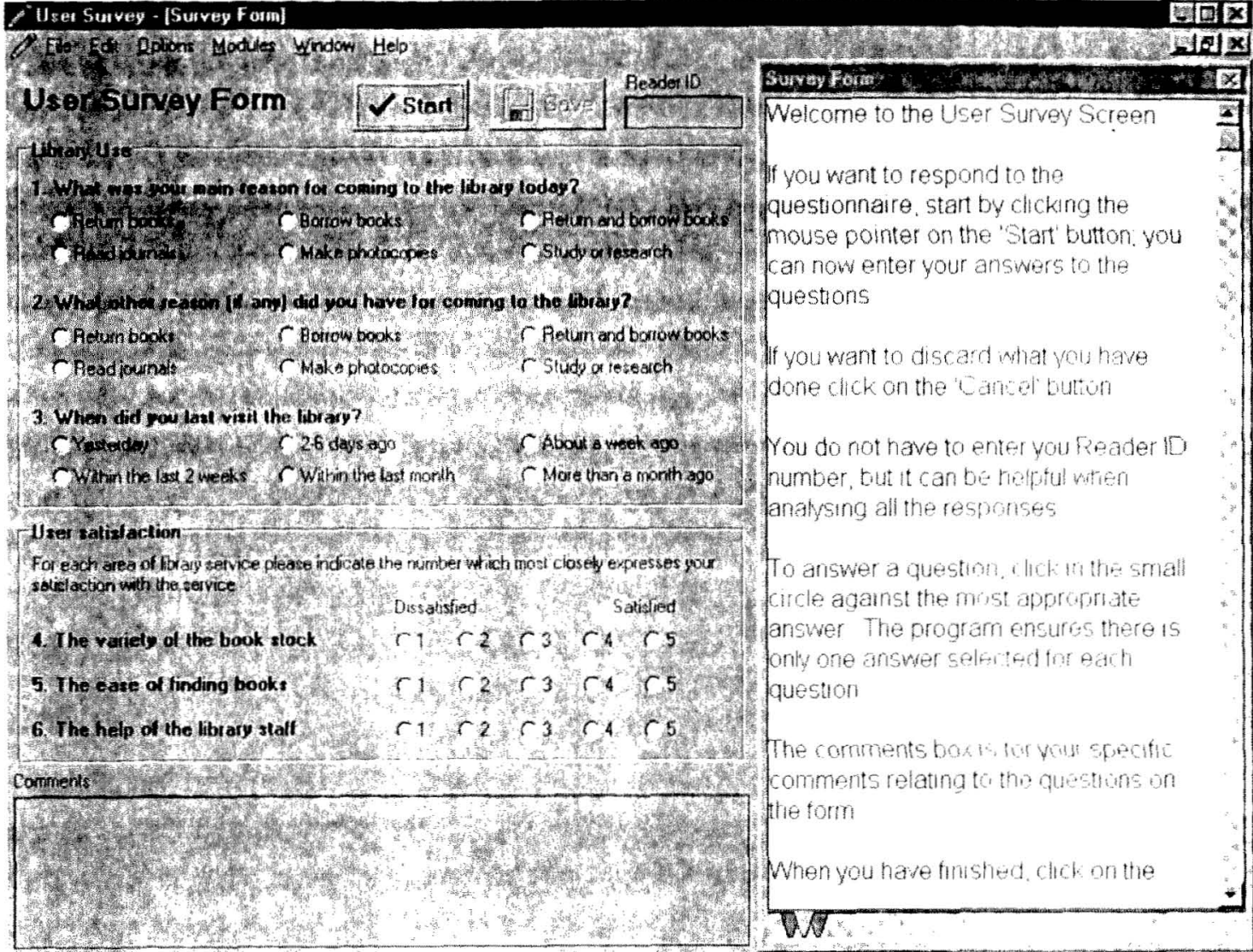

\section{Conclusiones}

Desde la perspectiva española, la investigación nos ha permitido constatar que las grandes diferencias existentes entre los sistemas bibliotecarios del Reino Unido, Italia y España no se ven reflejadas en la dinámica de gestión de las bibliotecas pequeñas y medianas. El perfil del profesional de este subsector corresponde a alguien que trabaja de forma muy independiente, toma sus decisiones basándose en la propia experiencia y la consulta a otros bibliotecarios del sector, está más interesado en los datos de satisfacción de los usuarios respecto al fondo, servicio e instalaciones de la biblioteca que en lo relativo al presupuesto, y no utiliza aplicaciones informáticas para la gestión del centro.

Asimismo se han puesto en evidencia las diferencias entre los distintos tipos de bi bliotecas incluidos en la investigación y su distanciamiento de las grandes bibliotecas universitarias. La exigencia desde la Comisión Europea de usar la tabla de medidas e indicadores de De Monfort University, obtenida en un proyecto anterior en el marco de bibliotecas universitarias, ha demostrado que una gran parte de estos datos no ofrece ningún interés para los centros pequeños. Posiblemente un cuestionario diseñado a medida habría proporcionado resultados más útiles para su aplicación al módulo. 
El desarrollo del proyecto ha permitido constatar cuáles son los ámbitos de responsabilidad que asumen los responsables de bibliotecas pequeñas y medianas, mostrando los tipos de decisión más extendidos y, en consecuencia, los indicadores y medidas por los que están más interesados. Sin embargo, el auge actual de la medición del rendimiento no parece haber convencido a los responsables de centros de tamaño pequeño y medio. El uso de datos estadísticos en la gestión responde más a menudo a la presión política de la organización o a la necesidad de justificar su actuación, que a una auténtica convicción de su utilidad.

A través del descubrimiento de las capacidades del módulo desarrollado, los responsables de bibliotecas han comprobado que el uso de una herramienta de soporte les permitirá ampliar la gama de datos e informaciones que actualmente utilizan en la toma de decisiones y podrán hacer un uso mayor de los datos estadísticos, siempre y cuando el módulo reúna unas condiciones de flexibilidad, adaptabilidad, bajo coste, integración y facilidad de uso que se requieren en un entorno tan diversificado y cambiante como el de los centros pequeños y medianos.

A pesar de la presión de los plazos marcados por la Comisión, los retrasos en el cobro de las cantidades subvencionadas y el esfuerzo complementario de la traducción de los materiales no contemplado por la Comisión, nuestro balance del proyecto es altamente positivo, debido a la dinámica de trabajo entre los miembros del equipo, y al interés demostrado por las bibliotecas que han participado en el proyecto. Pero la mayor satisfacción ha sido ver los resultados de la investigación concretados en un producto útil, agradable y sencillo de manejar. Ahora sólo cabe desear que el camino restante hasta la comercialización siga la misma suerte y podamos disponer de un módulo de soporte a la toma de decisiones destinado a bibliotecas pequeñas y medianas.

\section{Bibliografía}

1. Flanagan, J. C. The Critical Incident Technique. Psychological Bulletin, 1954, 51 (4), p. 327-358.

2. McCLURE, C. R. Information for academic library decision-making: the case for organizational information management. Connecticut: Greenwood Press, 1980, (Contributions in librarianship and information science, 31 ).

3. WARD, S., et al. Library performance indicators and library management tools. European Commission DGXIII-E3 (EUR 16483 EN), 1995.

4. Abbott, Christine Performance measurement in library and information services. Londres: Aslib, 1994, (The Aslib know how series).

5. Claus per a l'èxit: indicadors de rendiment per a biblioteques públiques. Barcelona: Eumo: Diputació de Barcelona: Col.legi Oficial de Bibliotecaris-Documentalistes de Catalunya, 1995, (Materials per a la biblioteca pública, 1).

6. International Standards Organization. Information and Documentation: library performance indicators. ISO (draft version), 1995.

Documentos del proyecto DECIMAL que pueden consultarse en línea:

Integrative Research Report. [en línea] Fisher, Shelagh; Mercè Bosch; Constança Espelt et al. LIB-DECIMAL/4-3012 Decision making in libraries: decision research for 
the development of integrated library systems. http://www.mmu.ac.uk/h-ss/dic/research/irr/ index.htm.

User Requirements Specification. [en línea] Willson, Jonathan; Fisher, Shelagh; Siân Lambert; Tony Oulton; with Constança Espelt et al. LIB-DECIMAL/4-3012 Decision making in libraries: decision research for the development of integrated library systems.

http://www.mmu.ac.uk/h-ss/dic/research/usr/ index.htm 